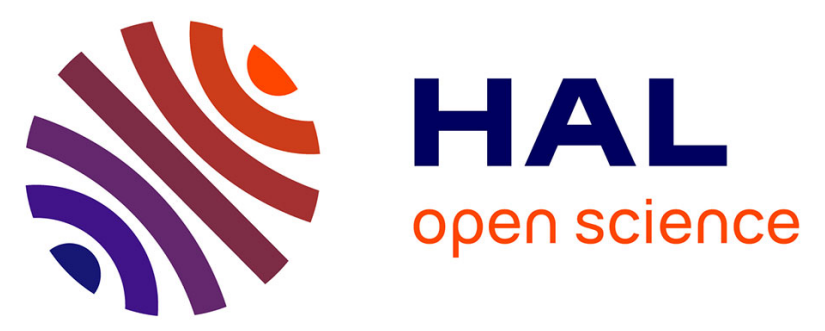

\title{
Simulation of Surface Resonant X-ray Diffraction
}

Yves Joly, Antoine Abisset, Aude Bailly, Maurizio de Santis, Farid Fettar, Stéphane Grenier, Danny Mannix, Aline y Ramos, Marie-Claire Saint-Lager, Yvonne Soldo-Olivier, et al.

\section{- To cite this version:}

Yves Joly, Antoine Abisset, Aude Bailly, Maurizio de Santis, Farid Fettar, et al.. Simulation of Surface Resonant X-ray Diffraction. Journal of Chemical Theory and Computation, 2018, 14 (2), pp.973 980. 10.1021/acs.jctc.7b01032 . hal-01707709

\section{HAL Id: hal-01707709 \\ https://hal.science/hal-01707709}

Submitted on 15 Feb 2018

HAL is a multi-disciplinary open access archive for the deposit and dissemination of scientific research documents, whether they are published or not. The documents may come from teaching and research institutions in France or abroad, or from public or private research centers.
L'archive ouverte pluridisciplinaire HAL, est destinée au dépôt et à la diffusion de documents scientifiques de niveau recherche, publiés ou non, émanant des établissements d'enseignement et de recherche français ou étrangers, des laboratoires publics ou privés. 


\title{
Simulation of surface resonant x-ray diffraction
}

\author{
Yves Joly, ${ }^{*}, \dagger$ Antoine Abisset, ${ }^{\dagger}$ Aude Bailly, ${ }^{\dagger}$ Maurizio De Santis, ${ }^{\dagger}$ Farid Fettar, $^{\dagger}$ \\ Stphane Grenier, ${ }^{\dagger}$ Danny Mannix, ${ }^{\dagger}$ Aline Y. Ramos, ${ }^{\dagger}$ Marie-Claire Saint-Lager, ${ }^{\dagger}$ \\ Yvonne Soldo-Olivier, $^{\dagger}$ Jean-Marc Tonnerre, ${ }^{\dagger}$ Sergey A. Guda, ${ }^{\ddagger}$ and Yvonne \\ Gründer
}

†Univ. Grenoble Alpes, CNRS, Grenoble INP, Institut Néel, 38042 Grenoble, France $\ddagger$ Institute for Mathematics, Mechanics, and Computer Science, Southern Federal University, 344090 Rostov-on-Don, Russia

ฯ Oliver Lodge Laboratory, Department of Physics, University of Liverpool, Liverpool, L69 7ZE, United Kingdom

E-mail: yves.joly@neel.cnrs.fr

\begin{abstract}
We present an ab initio numerical tool to simulate surface resonant x-ray diffraction experiments. The crystal truncation rods and the spectra around a given x-ray absorption edge are calculated at any position of the reciprocal space. Density functional theory is used to determine the resonant scattering factor of an atom within its local environment, and to calculate the diffraction peak intensities for surfaces covered with a thin film or with one or several adsorbed layers. Besides the sample geometry, the collected data also depend on several parameters, such as beam polarization and incidence and exit angles. In order to account for these factors, a numerical diffractometer mimicking the experimental operation modes has been created. Finally two case studies are presented in order to compare our simulations with experimental spec-
\end{abstract}


tra: i) a magnetite thin film deposited on a silver substrate, and ii) an electrochemical interface consisting of bromine atoms adsorbed on copper.

\section{Introduction}

Surface x-ray diffraction (SXRD) is widely used to solve the atomic structure at the surface of single-crystals. ${ }^{1}$ The use of synchrotron radiation is mandatory in order to get a sufficient sensitivity all along the crystal truncation rods (CTR), that is in between the bulk Bragg peaks perpendicularly to the investigated surface. One of its advantage is that in situ and even operando measurements can be performed, for example within an electrochemical environment or during a catalytic reaction. ${ }^{2,3}$ On the other hand, X-ray absorption near edge spectroscopy (XANES) is highly sensitive to the oxidation states considering the edge energy shift compared to a known reference. ${ }^{4}$ This sensitivity is even higher when using resonant x-ray diffraction (RXD). For instance RXD was used to quantitatively evaluate the charge ordering in the case of magnetite at low temperature ${ }^{5}$. Recording spectra across an energy range corresponding to an absorption edge is also possible in SXRD configuration. In this case, measurements are performed at specific points of the CTR, sensitive to the surface atoms to be probed and as in 3D diffraction, spectra exhibit large intensity variations around the edge. The so-called surface resonant x-ray diffraction (SRXRD) is therefore expected to be sensitive on both the surface and the electronic structure around the absorbing elements. For instance Chu et al. ${ }^{6}$ have observed the core-level energy shift associated to the oxidation state at the surface of Pt(111). Nevertheless, a thorough understanding of the recorded spectra has not been achieved yet and remains only qualitative. ${ }^{7}$ Menzel et al. used the FEFF ab initio simulation code to get the atomic form factors. Due to the lack of a complete simulation framework, they are calculated without accounting for their dependence on the momentum transfer, and additionally, non-equivalent absorbing atoms with their own geometrical surrounding and symmetry can not be considered. 
The SXRD data can be refined using existing fitting tools, such as ROD developed by E. Vlieg ${ }^{8}$. This program has been extended in order to include anomalous atomic form factors implying to record two sets of data: one far from any absorption edge and one at a specific edge. ${ }^{9}$ This increases the sensitivity on the surface parameters, especially the ones concerning the resonant atom. But it does not give access to the electronic parameters. Up to now, the rare simulations already performed ${ }^{7}$ did not include all the non-equivalent atoms or the complete dependence on the momentum transfer. This work aims at filling this lack and presents an ab initio way to simulate the corresponding spectra, as well as the CTR, acquired at different incident x-ray energies.

FDMNES ${ }^{10,11}$ is a self-consistent ab initio code, already extensively used to simulate XANES and RXD. Its Density Functional Theory (DFT) full potential approach makes it especially appropriate for simulating absorption edges of chemical elements embedded in non-close packed surroundings or in low symmetry sites. Relativistic calculations including spin-orbit give access to the heaviest chemical elements. Additionally, SRXRD, as XANES, is only poorly sensitive to the band gap or to the finest features specific to correlated materials. This all together makes the FDMNES code suitable for any kind of materials without restrictions. We present here a new capability of FDMNES, keeping the user-friendly philosophy. The experimental conditions must be easily reproduced in order to retrieve the relevant parameters governing the recorded signal. For this purpose the code now includes a virtual diffractometer mimicking the operation modes of most experiments. By this way, the beam polarization, incident and exit angles are automatically calculated for any value of the diffraction vector and all along the crystal truncation rods.

The paper is organized as follows: in section 2 the main theoretical elements of SRXRD are recalled. Section 3 is devoted to the implementation in the FDMNES code and to the characteristics of the calculation. We present in section 4, the virtual diffractometer. Section 5 presents two applications, showing the comparison between experimental data and simulated spectra of the Fe K-edge in magnetite on silver and K-edge of bromine on copper. 


\section{Surface resonant x-ray diffraction}

The theory of SRXRD is mostly common with the one of SXRD which can be found in review papers ${ }^{12,13}$; therefore in this section, we just recall its basic relations, with some focus related to the resonant extension and some practical specificity related to our technique of calculation. Because we want to perform simulations at crystal surfaces, but also for ultra thin films deposited on substrate, some overlap will occur with resonant x-ray reflectivity as done by Elzo, Grenier and collaborators in the Dyna code ${ }^{14}$, using a dynamical approach. Nevertheless remaining in the hard x-ray energy range, we keep the kinematic approximation, except for the damping inside the bulk discussed later. We neglect the refraction and consequently results at grazing incidence are not yet valid. This limitation will be overcome in the very near future. We now present the different components governing this technique.

\subsection{Bulk and Surface}

In surface crystallography it is convenient to separate the $2 \mathrm{D}$ unit cell between the bulk contribution where the atomic (and electronic) structure is supposed as in an infinite crystal and the surface which includes the substrate topmost atomic layers and the eventual adsorbed layers. In all this paper we thus call bulk, the semi-infinite part of the substrate keeping the non-distorted bulk geometry. The substrate contains thus the bulk, plus some layers possibly with relaxation or super-structure. Note that even with very weak geometrical distortions, the electronic structure of the topmost substrate layer is different from the ones deeper in the bulk. This implies a high level of anisotropy in the scattering amplitude, which must be calculated separately. The structure factor can thus be written as:

$$
F(\boldsymbol{Q}, \omega)=F_{\text {surf }}+F_{\text {bulk }}
$$

were $\boldsymbol{Q}=\boldsymbol{k}_{s}-\boldsymbol{k}_{i}$ is the momentum transfer, or scattering vector, $\boldsymbol{k}_{i(s)}$, being the momentum of the incoming (outgoing) photon beam. $\hbar \omega$ is the photon energy. The bulk structure factor, 
$F_{b u l k}$, is obtained by summing the structure factors of all the individual 3D unit cells, $F_{u}$, up to the infinity deep in the bulk. By this way we simply get:

$$
F_{b u l k}=\frac{1}{1-e^{-i Q \cdot c} \cdot e^{-\eta}} F_{u}
$$

where we use a bulk unit cell $(\boldsymbol{a}, \boldsymbol{b}, \boldsymbol{c})$ with $\boldsymbol{a}$ and $\boldsymbol{b}$ parallel to the surface, and $\boldsymbol{c}$ not necessarily perpendicular to it. The $e^{-\eta}$ term represents the wave damping between two unit cells, described later in section 3.3.

\subsection{Thin film}

When a film, thicker than typically 2 or 3 unit cell parameters is deposited on a substrate, the parametrization of all the inter-layer distances becomes pointless. More importantly, in our DFT approach, as shown in section 3, the calculation of the resonant form factor of all the non-equivalent absorbing sites becomes too time consuming, but also in fact unnecessary. Indeed, for an atom which is inside the film but at distances to both interfaces higher than typically 5 or $6 \AA$, its form factor is nearly identical to the one in a perfect bulk material. We thus have built the possibility to split the film in 3 parts. The first one contains the top most atomic layers of the film where all form factors are calculated in their 2D periodical environment. The second one is the inner part of the film and the 3D periodicity applies, thus the form factors are calculated as in a 3D bulk geometry. The third part is the interface between the film and the bulk which typically contains the film deepest and substrate topmost atomic layers. The form factors are calculated, as for the surface, using the local atomic geometry and not the 3D undistorted bulk one.

\subsection{Cap layer}

It is quite common to use a cap layer to protect the film from contamination (see example in section 5.1). This layer must be weakly adsorbed on the film, but it can happen that it 
is partially ordered. Fortunately this order is, most often incommensurate with the studied surface itself. It nonetheless can be seen in the specular reflection. We have thus also introduced this possibility in our calculations.

\subsection{Structure factor}

Including all terms considered here, the total structure factor can be written as:

$$
F=F_{\text {cap }}+F_{\text {surf }}+F_{\text {per }}+F_{\text {int }}+F_{\text {bulk }}
$$

where the subscripts cap, per and int stand respectively for the cap layer, the inner (periodical) part of the film, and the interface between film and bulk. Each of these contributions can be written as:

$$
F_{A}(\boldsymbol{Q}, \omega)=n_{A} \phi_{A}(\boldsymbol{Q}) \sum_{a} p_{a} r_{a} t_{a} e^{-i \boldsymbol{Q} \cdot \boldsymbol{R}_{a}}\left(f_{a}+f_{a}^{\prime}(\omega)-i f_{a}^{\prime \prime}(\omega)\right)
$$

where the index $A$ means any of the slabs cap, surf, per, int or bulk. a indexes the atoms, at position $\boldsymbol{R}_{a}$, in absolute unit, of $A$. $f_{a}$ is the non resonant form factor, sum of the Thomson term and the magnetic non resonant term. $f_{a}^{\prime}-i f_{a}^{\prime \prime}$ is the resonant, or anomalous, contribution. We have kept the common convention in x-ray diffraction: $f_{a}^{\prime \prime}>0$. It is seen as a simple correction in standard SXRD. It is, in this paper, the important term because it depends strongly on the photon energy. It also depends on the incoming (outgoing) beam polarizations, $\epsilon_{i(s)}$ when symmetry is not cubic as it is always the case in presence of surface or interfaces.

$p_{a}$ is the occupancy rate of the atom $a$. For the substrate, the film and the cap layer, surfaces or interfaces roughness can be used. We have just coded the model corresponding to a Gaussian distribution of the terrace heights, which is equivalent to the occupancy rate

$r_{a}=0.5 \operatorname{erfc}\left(\frac{z_{a}-z_{0}}{\sqrt{2} \sigma}\right) . \quad \sigma$ is the root mean square roughness parameter, $z$ the direction perpendicular to the surface, $z_{0}$ the interface origin and $z_{a}$ the atom position. In practice, 
$F_{\text {bulk }}$ (Eq. 2) is calculated for a semi-infinite bulk with full occupancy. $z_{0}$ is set at the half of the inter-reticular distance from the topmost bulk layer. Then, the roughness is obtained first, by adding layers with $z_{a}>z_{0}$, giving $r_{a}<0.5$, second subtracting the atomic form factor weighted by the missing occupancy $\left(1-r_{a}\right)$ for the same number of layers but with $z_{a}<z_{0}$.

The effect of the thermal disorder is given by $t_{a}(\boldsymbol{Q})=e^{-0.5 \overline{u_{a}^{2}} Q^{2}}$, where $\overline{u_{a}^{2}}$ is the atomic mean square displacement.

It is possible to describe the different components with their own unit cell parameters. When they are different, it is possible to choose either the bulk or the film lattice parameters as reference $(r e f)$. When they are commensurate, the super-cell is automatically taken into account. The ratio between the surface of the $2 \mathrm{D}$ unit cell, of the reference, $S_{r e f}$, and of the considered slab, $S_{A}$, that is $n_{A}=S_{r e f} / S_{A}$, gives the correct relative weight, when summing all components. When the ratio is not rational, this number becomes zero when we are considering neither a diffraction peak nor a CTR from the reference unit cell, nor the specular reflection. $\phi_{A}(\boldsymbol{Q})=e^{-i \boldsymbol{Q} \cdot \boldsymbol{O}_{A}}$ is the phase factor associated to the chosen origin, $\boldsymbol{O}_{A}$, of the slab $A$. It is related to the inter-layer distance (in the 3 directions) between $A$ and the slab just below.

\section{$3 \quad A b$ initio calculation}

\subsection{FDMNES}

The specificity of SRXRD versus SXRD, is that the resonant form factors of the absorbing atoms strongly depend on the local environment, both geometrical and electronic. To retrieve useful information, it is better to calculate these terms following a first principle theory.

The FDMNES code is an ab initio software, which allows simulation of x-ray absorption spectroscopy as well as x-ray resonant and non-resonant scattering spectroscopies. One of its main characteristics is that two different techniques can be independently used to solve the 
electronic structure. The full potential finite difference method (FDM) precisely applies for arbitrarily shaped potentials ${ }^{15}$, while full multiple scattering theory (MST) gives rise to less precise but faster calculations. The calculations can be applied for all classes of materials and it is especially efficient for the K-edge of all elements and the $\mathrm{L}_{23}$ edges of the heavy ones. ${ }^{4}$ Note that a Time Dependent DFT (TDDFT) extension expands the scope of the software to the other edges ${ }^{16}$. Importantly a recent numerical development in FDMNES within the FDM framework ${ }^{17}$ makes this code up to 40 times faster than it was before. The response functions of most materials can thus now be computed on conventional personal computers.

\subsection{The resonant form factor}

Here we have to calculate the resonant atomic form factor:

$$
f^{\prime}-i f^{\prime \prime}=m \omega^{2} \sum_{n, g} \frac{\left\langle\varphi_{g}\left|\hat{o}_{s}^{*}\right| \varphi_{n}\right\rangle\left\langle\varphi_{n}\left|\hat{o}_{i}\right| \varphi_{g}\right\rangle}{\hbar \omega-\left(E_{n}-E_{g}\right)+i \Gamma / 2},
$$

where $g$ indexes the atomic core states whereas $n$ indexes the excited (or intermediate) states, above the Fermi level. $E_{g}$ and $E_{n}$ are their energies, $m$ is the electron mass and $\Gamma$ an energy dependent broadening related to the hole and intermediate state lifetimes. We have directly written the formula within a mono-electronic approach avoiding thus a summation over all the electrons of the system. When considering only the electric transition up to the quadrupolar term, the transition operator is given by $\hat{o}=\epsilon \cdot \mathbf{r}+\frac{i}{2}(\epsilon \cdot \boldsymbol{r})(\boldsymbol{k} \cdot \boldsymbol{r})$, where $\boldsymbol{r}$ is the position. In Eq. 5, the index on the operator recalls that the incoming and outgoing polarizations and wave vectors are in general different. Their orientation in the sample frame results from the chosen operation mode as seen in section 4 .

What is calculated using the first principle approach are the photoelectron states $n$ in the formula above. Whatever the method, FDM or MST, FDMNES uses a cluster approach. In this one, the absorbing atom is, by default, set at the center of the cluster of atoms according to its surrounding geometry and with a radius typically up to 5 to $7 \AA$. Inside this cluster the 
electronic structure is solved, possibly in self-consistently way, then a last step calculates the $n$ states from the Fermi level up to the energy covered by the experiment. The calculation of Eq. 5 is then fast and direct. This procedure is repeated for all the non equivalent absorbing atoms of the 2D unit cell. The core level energies of all the atomic sites being calculated, the spectra are calculated versus the photon energy and not the photoelectron kinetic energy, giving thus sensitivity on the edge shifts related to the different oxidation states.

\subsection{Self absorption in the bulk}

As seen from Eq. 2, one needs to calculate the self absorption in the bulk, taking into account the incoming and outgoing beam absorption, and given by $e^{-\eta}$ where $\eta$ is:

$$
\eta(\omega)=\cos \left(\gamma_{z}\right) c\left(\frac{\mu_{i}(\omega)}{\sin (\alpha)}+\frac{\mu_{s}(\omega)}{\sin (\beta)}\right)
$$

where $\gamma_{z}$ is the angle between the normal to the surface and $\boldsymbol{c} . \mu_{i}$ and $\mu_{s}$ are the linear absorption coefficients corresponding to the incoming and scattered beams. When the bulk is not cubic or when we consider the quadrupolar term, they are most often different. Anyway, at this stage it is sufficient to take their spherical average.

When there is no atom of the calculated edge in the bulk, this scheme can be simplified, and the linear absorption coefficient can be taken as isotropic and constant. Otherwise, this one is calculated following our DFT procedure. It is important because its value at the edge changes dramatically, and consequently the sensitivity on the bulk itself strongly decreases when the absorption is maximum, typically at the white line just after the edge.

\subsection{Intensity calculation}

The final intensities $I(\boldsymbol{Q}, \omega)=\left|F / S_{\text {ref }}\right|^{2}$ are then calculated according to the equations 2, $3,4,5$, and 6 , including both resonant and non resonant atoms, and using the polarization conditions resulting from the different operation modes. Calculations can be performed 
using only a bulk and some surface layers, or using a thicker film as stated above. Special outputs to get both the CTR at any energy or the spectra at any $L$ value of the CTR are offered. Note also that FDMNES allows the comparison of data and calculated spectra using R-factor. A complete fitting procedure is nevertheless still in progress.

\section{Virtual diffractometer}

The operator $\hat{o}$ of the resonant atomic form factor in Eq. 5, depends on the polarization and wave vector direction of both the incoming and scattered waves. The self-absorption in the bulk related to the $\eta$ factor in Eq.2 depends on paths of the beam, that is on the incidence and exit angles. To perform the simulation of SRXRD, one thus needs to know all these vector directions and angles at any value of the scattering vector, that is at any point on the truncation rods.

Furthermore, the experiments are performed using different diffractometer types with $3,4,5$ or 6 circles, each of them bringing one of the angles governing the position of the sample or of the detector. A series of papers has demonstrated the formulas giving all these angles: Busing and Levy for the classical 3 and 4 circle $^{18}$, followed by systems specifically designed for surface crystallography as the one by Evans and Lutterodt for the so-called '2+2' diffractometer ${ }^{19}$, E. Vlieg and coworkers for a $5^{20}$, and Lohmeier and Vlieg for a 6 circle diffractometer ${ }^{21}$. Specific geometry often used in surface diffraction as the $z$-axis mode has been studied by Mati Block ${ }^{22}$ and finally H. You ${ }^{23}$ did a study concerning also up to the 6-circle diffractometer, but in fact general. This paper also contains a table specifying in a general way the possible operation modes. This same table, slightly modified, was further implemented by the authors of the (C)Spec software ${ }^{24}$ driving many of the diffractometers used widely. Because most users use the operation modes defined by this table, in our coding, we have followed You's relations given in his paper, amended for some typo mistakes and extended for not studied cases. These relations give the diffractometer angles, but also the 
incidence and exit angles and the direction of the polarizations and wave vectors.

We will not remake here the demonstration which can be found in You's paper but this one is obtained making the equality between the scattering vector and the reciprocal space vector, $\boldsymbol{H}$, for a given orientation by the diffractometer rotation circles, in the laboratory frame where $\epsilon_{i}$ and $k_{i}$ are known:

$$
\boldsymbol{Q}=Z U B \boldsymbol{H}
$$

where $B$ is the matrix to write $\boldsymbol{H}$ in Cartesian coordinates, $U$, the orientation matrix, which relates the crystal Cartesian coordinate system to the laboratory frame and finally $Z$ is the product of all rotation matrices. From this plus 3 chosen conditions on the angles, all the other angles can be calculated. These chosen conditions on the angles are called operation modes. The You's table, modified in (C) Spec, allowing their definition is:

Table 1: Diffractometer angles or condition on the angles governing the possible operation modes. The choice of 3 of them in 3 different columns defines the operation mode.

\begin{tabular}{cccccc}
\hline & Detector & Reference & Sample & Sample & Sample \\
\hline 1 & $\delta$ & $\alpha=\beta$ & $\eta$ & $\eta$ & $\eta$ \\
2 & $\nu$ & $\alpha$ & $\mu$ & $\mu$ & $\mu$ \\
3 & $Q_{z}$ & $\beta$ & $\chi$ & $\chi$ & $\chi$ \\
4 & $N_{z}$ & $\psi$ & $\phi$ & $\phi$ & $\phi$ \\
5 &. &. & $\eta=\delta / 2$ & $\eta=\delta / 2$ & $\eta=\delta / 2$ \\
6 &. &. & $\mu=\nu / 2$ & $\mu=\nu / 2$ & $\mu=\nu / 2$ \\
\hline
\end{tabular}

In the table, $\delta$ and $\nu$, are the polar and azimuthal angles of the detector, $\mathrm{Q}_{z}$ and $\mathrm{N}_{z}$ are the azimuthal rotation angles of the scattering vector and of the normal with respect to the sample surface in the laboratory reference frame. $\alpha$ and $\beta$ are the incidence and exit angles to the surface, $\psi$ is a pseudo angle giving the sample azimuth around the scattering vector. $\phi, \chi, \mu$ and $\eta$ are the 4 sample rotation angles. A figure showing all these angles can be found in You's paper. The operation mode is done by fixing 3 of these angles or conditions on these angles, taking them in 3 different columns of the table 1 .

It is important to note that many setups use in fact only 4 circles. This means that 2 
angles are imposed by construction and the freedom on the operation modes concerns thus in practice only one condition, very often chosen in the Reference column of the table. For example in the 4-circle z-axis mode diffractometer, $\chi$ is fixed to zero and thus, the sample is vertical in the laboratory frame. Consequently, the sample, $\mu$, and detector, $\nu$, rotation circles, not stacked in the same way, give directly the incidence and exit angles $\alpha$ and $\beta$. Another consequence is that $\phi$ is by convention fixed to zero because it has the same rotation axis than $\eta$ and becomes redundant, except for the correction of small misalignment. Note also that, in this mode, the incoming polarization is usually $\pi$, that is in the incidence plane.

The interest for us is that using this way, as stated above, we recreate the corresponding experimental conditions, and thus the orientations of the vectors $\boldsymbol{k}_{i}, \boldsymbol{k}_{s}, \epsilon_{i}, \epsilon_{s}$ in the sample frame, and the angles $\alpha$ and $\beta$. In addition, the obtained diffractometer angles allow the comparison of their values with the experiment and thus to check that all is consistent.

\section{Application}

\subsection{Magnetite thin film}

At $T_{V} \approx 120 \mathrm{~K}$, magnetite undergoes a metal-insulator transition explained by a charge ordering phenomenon. ${ }^{25}$ The context of this study concerns the searching of this transition for a very thin film, grown by molecular beam epitaxy on $\operatorname{Ag}(001)$. In this paper we nevertheless just want to give an example of our simulation technique in a complex system and we analyze thus only the data recorded at $130 \mathrm{~K}$, that is above $T_{V}$. The film thickness is supposed to be about $74 \AA$, that is 8.75 unit cells of the room temperature phase. A gold cap layer covers the film in order to protect it. The SRXRD measurements were conducted at BM02 (D2AM), a French-CRG beamline at the ESRF, Grenoble.

The film grows epitaxially, (001) oriented and with unit cell parameters incommensurate with the substrate. It presents a tetrahedral distortion from the room temperature (RT) $3 \mathrm{D}$ cubic phase, where $a=8.394 \AA$. Indeed we found $a=b=8.29(1) \AA$ and $c=8.454 \AA$. 
We used these $\mathrm{Fe}_{3} \mathrm{O}_{4}$ unit cell parameters as reference for the reciprocal space indexation. For the Ag substrate, we found $a=4.0675(40) \AA$, thus slightly contracted as it must be, compared with its RT value. Fig. 1 shows the specular truncation rod, recorded at $130 \mathrm{~K}$ and at $7112 \mathrm{eV}$. Experiments were performed for this reflexion using the so-called 242 mode that is following the table 1 , with $\nu=0, \psi=0$ and $\mu=0$. Polarization is thus parallel to the surface. Dependence on the azimuth (given by $\psi$ ) is in this case very small but at $L=2$, not considered here. It is corrected to take into account the sample surface illuminated by the x-ray beam and the small slit width. We observe the film Kiessig fringes related to the film thickness as in traditional reflectivity. The growth along the $\mathrm{Fe}_{3} \mathrm{O}_{4}(001)$ direction is revealed by its $Q=(004)$ Bragg peak, while the $\operatorname{Ag}(002)$ stands at $L=4.14$. At about $L=3.5$, a broad $\mathrm{Au}(111)$ reflection can be seen, showing that the cap layer presents some small correlation length along this direction. It nevertheless presents no of its own Kiessig fringes, and thus a very large roughness, potentially with a 3D morphology. It is indeed known that $\mathrm{Au}$ forms 3D clusters on magnetite in this thickness range. ${ }^{26}$ Moreover we also found that the fringes mainly correspond to the magnetite which is of high quality.

To calculate the spectra it is mandatory to have first a satisfactory accord on the CTR, because it gives the contribution on the scattered amplitude of the substrate and of the cap layer. We used $5 \AA$ cluster radius and the Multiple Scattering Theory to get the Fe form factors. Larger radius and FDM usually give better agreement but the number of unknown structural parameters makes for the moment more precise calculation non useful. In such system it is quite complex, because the morphology of the cap layer is itself rather unknown. In order to get the agreement shown in Fig. 1, we used a $20 \AA$ cap thickness but with a 0.18 occupancy rate and a $8 \AA$ root mean square roughness at the Au/vacuum interface. The distance between top most magnetite layer and bottom most gold layer is $2.7 \AA$. To describe the magnetite film we used a simple periodical model without interface and surface slabs. The corresponding film is found to be $71.2 \AA$ thick at $2.4 \AA$ from the Ag substrate and with a $2 \AA$ root mean square roughness at its interface with gold. The roughness at the 
film/Ag interface is small $(0.8 \AA)$ but mandatory to get the resulting agreement, specially between $\mathrm{L}=0.5$ and 3.5 where the resulting decrease of $F_{\text {bulk }}$ is the stronger. We also found a sensitivity on the phase factor of the film versus the Ag substrate one and we found a better agreement when the bottom most atom of the film is a Fe octahedral site than when it is an Fe tetrahedral site or an oxygen site. We nevertheless think that the data set is not sufficient to give a full description of the $\mathrm{Fe}_{3} \mathrm{O}_{4} / \mathrm{Ag}$ interface.

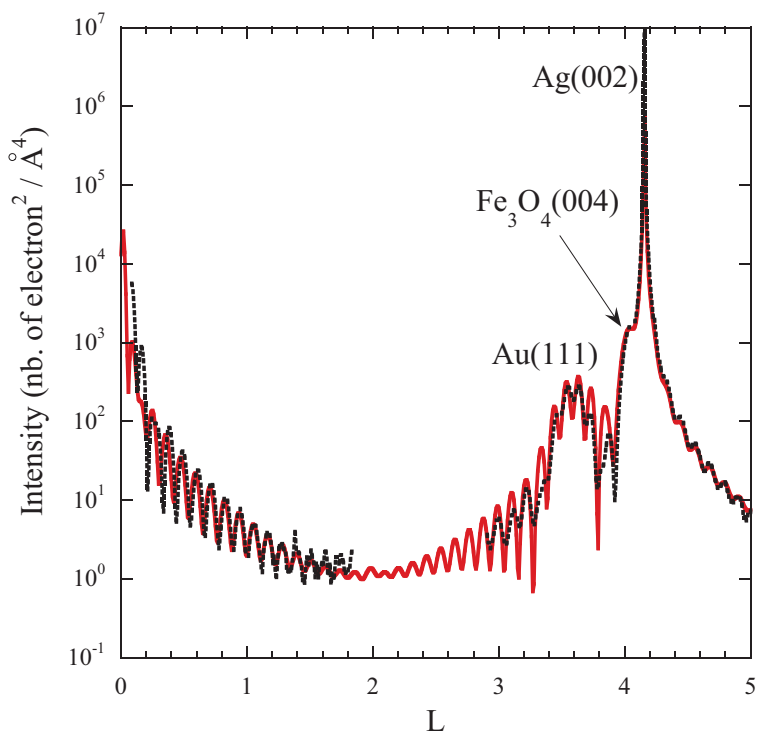

Figure 1: Crystal truncation rod of the specular reflection in $\mathrm{Fe}_{3} \mathrm{O}_{4}$ on $\mathrm{Ag}(001)$. The photon energy is $7112 \mathrm{eV}$ and the polarization is perpendicular to the incident plane. The $L$ index is calculated using the magnetite unit cell parameters. Black dots are the experimental data corrected to take into account the slit width, and the red full line is the simulation. The Au cap layer being partially ordered participates to the signal around $L=3.5$.

In Fig. 2, we show the spectra measured at $L=1$ and $130 \mathrm{~K}$ and the fluorescence signal. The simulations were performed using the usual RT phase but tetrahedraly distorted as stated above. We clearly see the main features of the iron K-edge in magnetite despite the noisy and low intense signal. The (001) reflection is forbidden in the RT space group. Thus magnetite signal, at this $L$ value, comes from the atomic layers which are not cancelled out, due to the roughness at both magnetite interfaces, and by the remaining un-complete 0.42 unit cell, on top of the 8 complete ones and corresponding to a $3.568 \AA$ magnetite slab. Another possibility can be conjectured: the strain decreases more the symmetry than the 
simple tetrahedral transformation, making the (001) reflection not anymore forbidden even above $T_{V}$. Note also that in the (001) data, the fluorescence being far less intense than the scattering cross section, the increase at the energy edge cannot be attributed to this phenomenon.

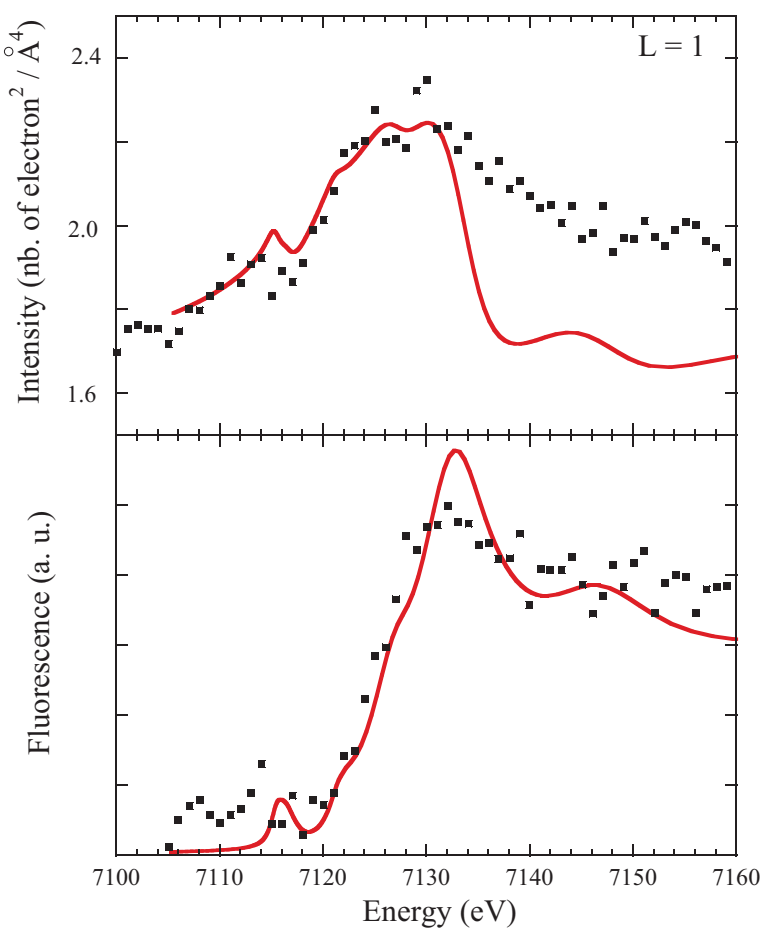

Figure 2: Fluorescence (bottom) and $Q=(001)$ SRXRD spectra (top) around the Fe K-edge in $\mathrm{Fe}_{3} \mathrm{O}_{4}$ on $\mathrm{Ag}(001)$. Black dotted line is the experiment, red full line is the simulation. Despite the very low signal at the forbidden $L$ value, the signal is mainly reproduced.

Our most important result is that we are able to simulate both resonant spectra and truncation rods in a very complex system. The agreement on the spectra is not excellent in this case, the full structure being not well known and the data itself being not very well resolved. Better reproduction of the spectra could nevertheless be obtained using the interface and surface options as proposed in section 2.2. This will also need a larger data set with non-specular CTR and more importantly a large number of spectra recorded at different $L$ values on the CTR. Next section show a better agreement on another system. Nevertheless we open the possibility of the analysis of data recorded at temperatures below the Verwey transition and thus the way, using simulations, to verify the charge ordering 
phenomena observed in bulk magnetite.

\subsection{Bromine on copper}

The context of this study concerns the probe of charge transfer and nature of the chemical bonding at an electrochemical interface. ${ }^{27} \mathrm{Br}$ adsorption onto $\mathrm{Cu}(001)$ is an ideal system for attempting SRXRD measurements, as the Br halide anions form simple ordered $\mathrm{c}(2 \mathrm{x} 2)$ adlayers on the $\mathrm{Cu}(001)$ surface with adsorption uniquely into the 4-fold $\mathrm{Cu}$ hollow site ${ }^{28,29}$. The data were recorded in situ at the BM28 (XMaS), the UK-CRG beamline at the ESRF, Grenoble. The $(0,1,0.2)$ position in reciprocal space which corresponds to the signal of the $\mathrm{c}(2 \mathrm{x} 2)$ superstructure induced by adsorption of the bromine was recorded at the Br K-edge in the $\mathrm{Cu}(001)$-halide electrochemical systems. The $(1,1,0.2)$ position was probed for the $\mathrm{Cu} \mathrm{K}$-edge, as this is the most surface sensitive position of the CTR. The measurements performed with polarization parallel and perpendicular to the scattering plane, show strong differences giving thus a clear sensitivity on the local electronic structure (Fig. 3).

For the simulation we have used the atomic positions and mean square displacements from Saracino and coworkers ${ }^{28,29}$. At this stage, we have performed a self-consistent simulation to get the electronic structure and calculated the unoccupied states to obtain the form factors, using the finite difference method. This point is mandatory to get a satisfactory agreement

in this kind of system where symmetry is very low. The cluster radius was fixed to $6 \AA$ for both steps. This simple approach gives a good agreement for both polarizations and at both edges without any further optimization.

Though the comparison is difficult between studies on different compounds and at different edges, the agreement between the data and the model presented in Fig 3 is clearly better than the one found by Menzel et al. ${ }^{7}$ using partly an ab initio procedure. On the contrary the agreement obtained by Chu and coworkers ${ }^{6}$ is very good. Nevertheless their study is not $a b$ initio, the atomic form factor was deduced from the fluorescence spectra and the oxidation of the metal was mimicked by a simple shift in the energy scale corresponding 


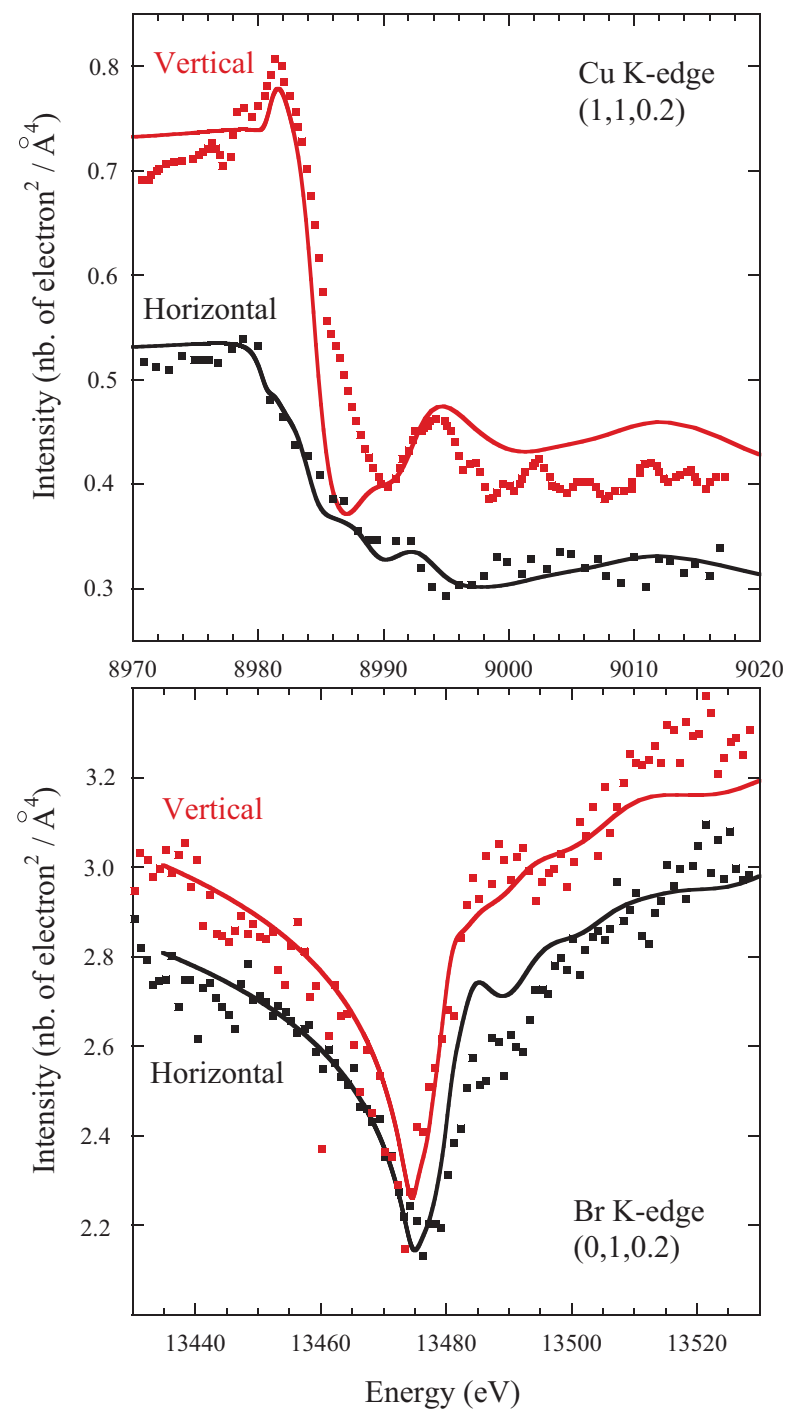

Figure 3: SRXRD spectra at the $\mathrm{Br}$ (bottom, $Q=(0,1,0.2)$ ) and $\mathrm{Cu}$ (top, $Q=(1,1,0.2)$ ) $\mathrm{K}$-edges in the $\mathrm{Br} \mathrm{c}(2 \times 2)$ adlayer on $\mathrm{Cu}(001)$. The linear dichroism is strong and show the sensitivity on the local electronic structure. The agreement between simulation (full line) and experiment (dotted) is good on both polarizations and at both absorption edges. 
to a change in the bounding energy. This shift was found to be $9 \mathrm{eV}$ which is higher than typical values in metal oxides.

The ab initio simulation includes the calculation of the electronic states around the absorbing atoms. Their projection can be obtained, resolved in $\ell$ and $m$, the quantum number of the spherical harmonics, inside the atoms. To illustrate this, we show in Fig. 3 the density of states (DOS) integrated in the Br atom as well as in the different non equivalent copper atoms. Only the energy range below the Fermi level is shown; above the Fermi level the $p$-DOS is nearly proportional to the imaginary part of the form factor. $\mathrm{Br}$ presents a peculiar feature, mainly $4 p_{x}$ and $4 p_{y}$ below $-7 \mathrm{eV}$. More importantly we can see the correspondences of several peaks at the same energy as -2.80 and $-2.68 \mathrm{eV}$ between the $\mathrm{Br}$ and the copper atoms in layer $1\left(\mathrm{Cu}_{1}\right)$ and layer $2, \mathrm{Cu}_{2 a}$ which is in the 2nd atomic Copper layer below the $\mathrm{Br}$ atom, and $\mathrm{Cu}_{2 b}$, which is in the 2nd atomic copper layer below an unoccupied hollow site. We have also plotted the $3 d_{z^{2}}$ and $4 p_{z}-\mathrm{Cu}$ states and the $4 p_{z}-\mathrm{Br}$ states to check the hybridization and band effect on these peculiar projections. The peak at $-4.6 \mathrm{eV}$ in $\mathrm{Cu}_{2 a}$ and $\mathrm{Br}$ reveal the hybridization $3 d_{z^{2}}-4 p_{z^{-}}-\mathrm{Cu}_{2 a}$ giving a slight electric moment on this atom toward the apical Br. At the same energy a peak in the $4 p_{z}$-Br states is found which reveals a specific bonding. Because it is a de-localized state, it can also be seen in the other components of the $3 d$ states of $\mathrm{Cu}_{2 b}$. One can also verify that at the second $\mathrm{Cu}$ layer, surface states are still important, the DOS being not yet similar to the Cu bulk atom ones.

FDMNES does not give Mullikan analysis of the orbital occupancy, but the integration up to the atomic radius is nevertheless a good indication of it for the localized orbital. We found a $3 d^{9.7}$ configuration for $\mathrm{Cu}_{1}$. The results for the other copper atoms are close but to have a more realistic convergence, we would have to include the effect of the disordered counter-ions, the so-called Helmholtz layer above the Br atoms. The interest of this approach is that when the fitting of the structural parameter by comparison to the data is good, the associated DOS must also be correct. In this way, one gets at the same time the geometric structure and the electronic structure of the investigated surface, which opens up the possibility to obtain 


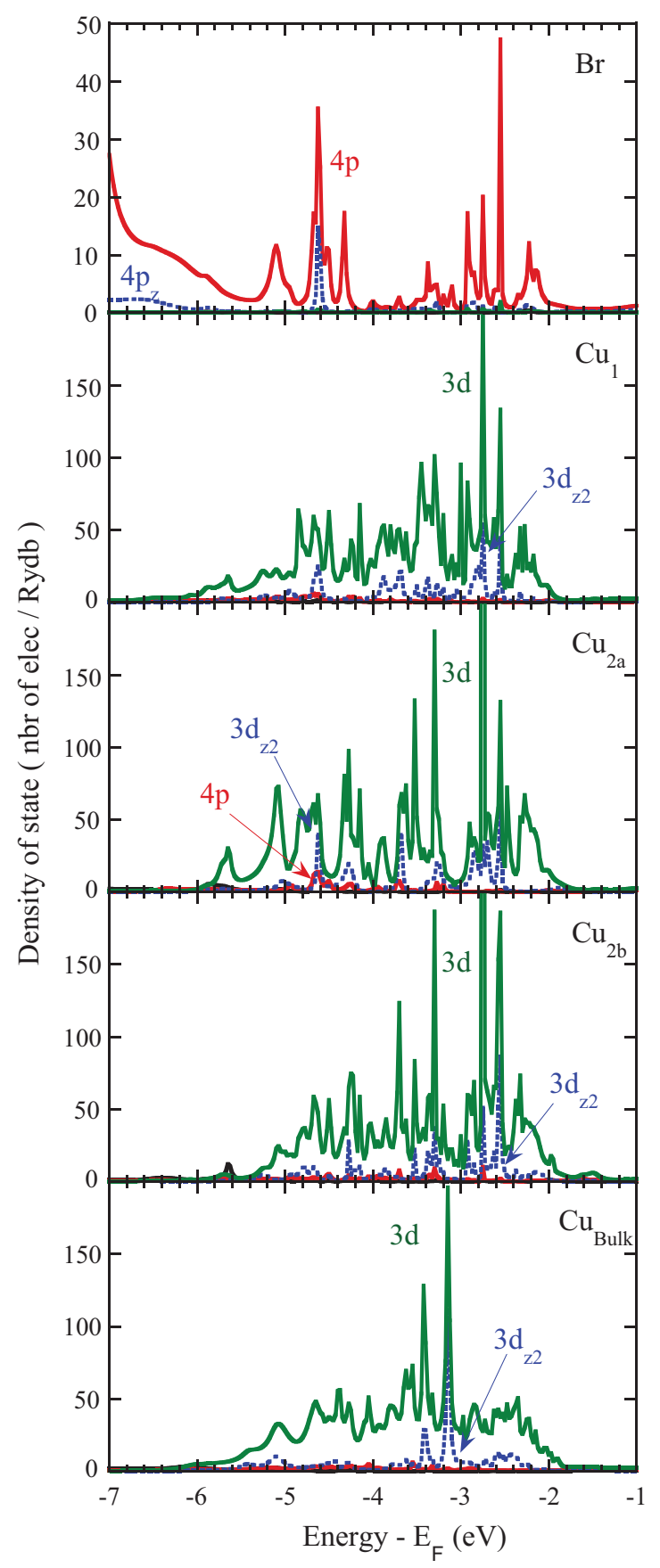

Figure 4: Density of state of the non equivalent atoms, from the $\mathrm{Br}$ (top), to the top most $\mathrm{Cu}_{1}$, the second layer $\mathrm{Cu}_{2 a}$ and $\mathrm{Cu}_{2 b}$ and the bulk copper, $\mathrm{Cu}_{\text {Bulk }}$. This scheme illustrates the surface states in the top most $\mathrm{Cu}$ and Br layers, before converging to the bulk copper states. The origin of the energy scale is the Fermi energy. 
detailed insight into the bonding at electrochemical surfaces and for surfaces under reactive condition which do not allow for UHV spectroscopic studies.

\section{Conclusion}

We have presented a new tool allowing the ab initio simulation of surface resonant $\mathrm{x}$-ray diffraction. This one, written to be user friendly allows the simulation of both the crystal truncation rods and the spectra at any point on them. This has been included in the FDMNES code which allows full potential calculation and thus to be very precise for calculation at the K-edge of all elements and at the $\mathrm{L}_{23}$ edges of the heavy ones. Being also adapted to magnetic calculation, many applications can be considered. The TDDFT approach included in FDMNES could also allow such simulations at the $\mathrm{L}_{23}$ edges of $3 d$ transition metal atoms. Some extensions still need to be included as for example easy fit procedure on the crystallographic parameters, non-spherical Debye model for the surface atoms, or dynamical aspects giving the refraction effect at the interfaces. This last point can be important when working at grazing incidence, close to the critical angle. Around an edge, it is even possible that the refraction index value can be greater or less than 1 . In the case of electrochemical interface, we can also add that a completely satisfactory evaluation of surface atoms valence state must need the taking into account of the Helmholtz layer. Nevertheless we have already shown in this paper that our approach works for the study of two very different systems: a thin magnetite film on silver and a Br layer adsorbed on copper in an electrochemical interface.

\section{Acknowledgement}

S.A. Guda thanks the Russian Ministry of Education and Science for the financial support (project RFMEFI 58716X0027, agreement no. 14.587.21.0027). Y. Gründer thanks the Royal Society for funding through a University Research Fellowship. 


\section{References}

(1) Robinson, I. K. Crystal truncation rods and surface roughness. Phys. Rev. B 1986, 33, 3830.

(2) Gründer, Y.; Lucas, C. A. Surface X-ray diffraction studies of single crystal electrocatalysts. Nano Energy 2016, 29, 378 - 393, Electrocatalysis.

(3) Saint-Lager, M.-C.; Laoufi, I.; Bailly, A. Operando atomic structure and active sites of TiO2(110)-supported gold nanoparticles during carbon monoxide oxidation. Faraday Discuss. 2013, 162, 179-190.

(4) Joly, Y.; Grenier, S. Theory of absorption near edge structure. In X-Ray Absorption and X-Ray Emission Spectroscopy; Bokhoven, J. A. V., Lamberti, C., Eds.; John Wiley \& Sons, Ltd: Chichester, West-Sussex, United Kingdom, 2016; Chapter 4, pp 73-97.

(5) Nazarenko, E.; Lorenzo, J. E.; Joly, Y.; Hodeau, J.-L.; Mannix, D.; Marin, C. Resonant X-ray diffraction studies on the charge ordering in magnetite. Phys. Rev. Lett. 2006, 97, 056403.

(6) Chu, Y. S.; You, H.; Tanzer, J. A.; Lister, T. E.; Nagy, Z. Surface Resonance XRay Scattering Observation of Core-Electron Binding-Energy Shifts of Pt(111)-Surface Atoms during Electrochemical Oxidation. Phys. Rev. Lett. 1999, 83, 552-555.

(7) Menzel, A.; Chang, K.-C.; Komanicky, V.; You, H.; Chu, Y. S.; Tolmachev, Y. V.; Rehr, J. J. Resonance anomalous surface X-ray scattering. Radiation Physics and Chemistry 2006, 75, 1651 - 1660, Proceedings of the 20th International Conference on X-ray and Inner-Shell Processes.

(8) Vlieg, E. ROD: a program for surface X-ray crystallography. J. Appl. Cryst. 2000, 33, 401. 
(9) Vonk, V. Surface structure refinement including anomalous crystal truncation rods. Journal of Applied Crystallography 2011, 44, 1217-1221.

(10) Bunău, O.; Joly, Y. Self-consistent aspects of x-ray absorption calculations. J. Phys.: Condens. Matter 2009, 21, 345501.

(11) The code can be downloaded at the web address: www.neel.cnrs.fr/fdmnes.

(12) Feidenhans'l, R. Surface structure determination by X-ray diffraction. Surface Science Reports 1989, 10, 105.

(13) Robinson, I. K.; Tweet, D. J. Surface x-ray diffraction. Rep. Prog. Phys. 1992, 55, 599.

(14) Elzo, M.; Jal, E.; Bunau, O.; Grenier, S.; Joly, Y.; Ramos, A. Y.; Tolentino, H. C. N.; Tonnerre, J. M.; Jaouen, N. X-ray resonant magnetic reflectivity of stratified magnetic structures: Eigenwave formalism and application to a W/Fe/W trilayer. J. Magn. Magn. Mater. 2012, 324, 105.

(15) Joly, Y. X-ray absorption near-edge structure calculations beyond the muffin-tin approximation. Phys. Rev. B 2001, 63, 125120.

(16) Bunău, O.; Joly, Y. Time-dependent density functional theory applied to x-ray absorption spectroscopy. Phys. Rev. B 2012, 85, 155121, The code.

(17) Guda, S. A.; Guda, A. A.; Soldatov, M. A.; Lomachenko, K. A.; Bugaev, A. L.; Lamberti, C.; Gawelda, W.; Bressler, C.; Smolentsev, G.; Soldatov, A. V.; Joly, Y. Finite difference method accelerated with sparse solvers for structural analysis of the metalorganic complexes. J. Phys.: Conf. Ser. 2016, 712, 012004.

(18) Busing, W. R.; Levy, H. A. Angle calculations for 3- and 4-circle X-ray and neutron diffractometers. Acta Crystallographica 1967, 22, 457-464.

(19) Evans-Lutterodt, K. W.; Tang, M. T. Angle Calculations for a '2+2' Surface X-ray Diffractometer. Journal of Applied Crystallography 1995, 28, 318-326. 
(20) Vlieg, E.; Van der Veen, J. F.; Macdonald, J. E.; Miller, M. Angle calculations for a five-circle diffractometer used for surface X-ray diffraction. Journal of Applied Crystallography 1987, 20, 330-337.

(21) Lohmeier, M.; Vlieg, E. Angle calculations for a six-circle surface X-ray diffractometer. Journal of Applied Crystallography 1993, 26, 706-716.

(22) Bloch, J. M. Angle and index calculations for ' $z$-axis' X-ray diffractometer. Journal of Applied Crystallography 1985, 18, 33-36.

(23) You, H. Angle calculations for a '4S+2D' six-circle diffractometer. J. Appl. Cryst. 1999, 32, 614 .

(24) Inquiry on this code can be obtained at the web address: https://www.certif.com/content/contact/.

(25) Verwey, E. J. W. Electronic Conduction of Magnetite $\left(\mathrm{Fe}_{3} \mathrm{O}_{4}\right)$ and its Transition Point at Low Temperatures. Nature 1939, 144, 327-328.

(26) Spiridis, N.; Socha, R.; Handke, B.; Haber, J.; Szczepanik, M.; Korecki, J. Clustersupport interaction in AuFe3O4 system. Catalysis Today 2011, 169, 24 - 28.

(27) Gründer, Y.; Lucas, C. A. Probing the charge distribution at the electrochemical interface. Phys. Chem. Chem. Phys. 2017, 19, 8416-8422.

(28) Gründer, Y.; Kaminski, D.; Golks, F.; Krug, K.; Stettner, J.; Magnussen, O. M.; Franke, A.; Stremme, J.; Pehlke, E. Reversal of chloride-induced $\mathrm{Cu}(001)$ subsurface buckling in the electrochemical environment: An in situ surface x-ray diffraction and density functional theory study. Phys Rev B 2019, 81, 174114.

(29) Saracino, M.; Broekmann, P.; Gentz, K.; Becker, M.; Keller, H.; Janetzko, F.; Bredow, T.; Wandelt, K.; Dosch, H. Surface relaxation phenomena at electrified interfaces: 
Revealing adsorbate, potential, and solvent effects by combined x-ray diffraction, STM and DFT studies. Phys. Rev. B 2009, 79, 115448. 